\title{
Desenmascarando a los impostores: Los médicos profesionales y su lucha contra los falsos médicos en Perú
}

\author{
Unmasking impostors: Professional physicians and \\ their struggle against fake doctors in Peru
}

${ }^{1}$ Doctora en Historia. Investigadora, Departamento de Ciencias Históricas y Geográficas, Universidad de Tarapacá, Arica, Chile. $\bowtie$ ii

${ }^{2}$ Doctor en Historia. Profesor Asistente, Instituto de Historia, Pontificia Universidad Católica de Chile, Santiago, Chile. $\square$ iip
RESUMEN La caracterización de sanadores no-titulados como "charlatanes" o "impostores" ha influido notablemente en cómo han sido percibidos por la opinión pública y en las investigaciones académicas. Se creó, entonces, una división entre los médicos profesionales y aquellos que adquirieron su conocimiento de modo tradicional y noacadémico. Este artículo cuestiona la supuesta división entre dichos especialistas en el campo de la salud para ofrecer un cuadro más complejo y rico de prácticas locales a partir del caso peruano. A partir, sobre todo, de correspondencia de la Facultad de Medicina de Lima y de avisos en periódicos, reconstruimos la dinámica de las autoridades médicas en sus intentos, muchas veces infructuosos, de contener y excluir a sanadores de origen asiático, europeo o local. Para ello, estudiamos dos artefactos diseñados para legitimar y monitorear a los médicos formados profesionalmente: los títulos o diplomas y las listas de graduados, predecesores de nuestros modernos documentos de identidad y bases de datos.

PALABRAS CLAVES Historia de la Medicina; Licencia Médica; Medicina Tradicional; Identificación Social; Perú.

ABSTRACT The characterization of non-professional healers as "quacks" or "impostors" has influenced much of how such actors have been perceived by public opinion and in academic research. As a result of this, a divide has emerged between professional physicians, on the one hand, and those who acquired their knowledge in a traditional and non-academic way, on the other. This work questions the alleged divide between these two groups in the health field in order to offer a more complex and richer picture of local practices in Peru. Based mainly on correspondence from the Faculty of Medicine in Lima and newspaper ads, we reconstructed the attempts made by medical authorities to contain and exclude healers of Asian, European, or local backgrounds, many of which failed. For this reason, we studied two specific devices designed to legitimate and monitor physicians trained professionally: degrees or diplomas and lists of graduates, both of which are predecessors to our current identification cards and databases.

KEY WORDS History of Medicine; Licensure, Medical; Medicine, Traditional; Social Identification; Peru. 


\section{INTRODUCCIÓN}

En febrero de 2017, diversos medios de comunicación estadounidenses informaban del arresto de Malachi Love-Robinson, un adolescente residente en el estado de Florida acusado de practicar la medicina sin tener título profesional ni autorización para ello. En su defensa, Love-Robinson sostuvo que nunca pretendió ser médico y que si se hacía llamar "doctor" era porque poseía un doctorado en "divinidad", adquirido en internet por US\$29 dólares ${ }^{(1)}$. Más allá de una simple anécdota, el caso de Love-Robinson da cuenta de un grave problema de suplantación médica, que se repite con cierta regularidad en EEUU y América Latina. Por ejemplo, a mediados de 2018, el Colegio Médico de la Provincia de Buenos Aires denunció a un médico venezolano por suplantación de identidad y hacer uso de un título médico ajeno. En pocos días eran más de once los médicos extranjeros investigados por usurpación de títulos, honores y falsificación de documento público, que ejercían en centros de salud públicos y privados en la capital argentina ${ }^{(2)}$. Estas noticias demuestran que, no obstante los avances en el desarrollo de mecanismos de identificación, la práctica de falsos doctores en Latinoamérica continúa siendo muy difícil de erradicar.

Desde mediados del siglo XIX, América Latina atravesó un intenso proceso de profesionalización de la medicina y las disciplinas paramédicas, con el objetivo de consolidar una identidad de grupo en la que fue decisiva la supresión de sanadores no titulados del mercado medicinal ${ }^{(3,4,5)}$. Hasta hace algunas décadas, la historia tradicional de la medicina había reducido a la categoría de "charlatanes" a quienes brindaban atención médica fuera del campo diplomado de la salud, siendo acusados reiteradamente de atentar contra la salud de los pacientes. Sin embargo, en los últimos años, nuevas investigaciones han analizado la complejidad de estos sanadores y médicos sin licencia capaces de usar posturas, prácticas y terminologías propias de la medicina oficial| ${ }^{(6,7)}$. Como ha planteado el historiador Diego Armus, existió una suerte de zona gris caracterizada por la superposición de saberes médicos, un área que no era de legítima competencia de los médicos profesionales y que necesita ser más estudiada por parte de los investigadores ${ }^{(8)}$. Precisamente, este complejo escenario de saberes hace muy difícil encontrar un término único para definirlos, pues abarca desde quienes habían pasado por las aulas universitarias sin haberse graduado hasta los que simplemente buscaban enriquecerse a costa de la desesperación de los pacientes o como producto de un conocimiento heredado o adquirido a través de prácticas ancestrales. Para fines de este artículo, utilizaremos el término "sanadores no titulados" para referirnos a este grupo en particular, que opera fuera del radio de influencia institucional de la Facultad de Medicina. Al hacerlo, buscamos alejarnos del contenido peyorativo de términos usados en documentos oficiales (y en el lenguaje popular) como "charlatanes".

Siguiendo estas nuevas aproximaciones, este artículo analiza las diversas estrategias y discursos esgrimidos por los médicos profesionales en Perú reunidos en torno a la Facultad de Medicina de Lima - la única existente en el país hasta 1935 - para diferenciarse de otros sanadores no profesionales. La metodología del presente ensayo combina dos áreas en particular: los estudios sobre vigilancia e identificación y la historia de la salud. La intersección de ambas perspectivas sugiere nuevas formas de estudiar el proceso de profesionalización de la medicina desde la generación y circulación de determinados mecanismos orientados a desarrollar identidades documentadas. Como buscamos demostrar en las siguientes páginas, los médicos profesionales en Perú introdujeron nuevas técnicas, sistemas y prácticas con el propósito de establecer un monopolio de profesionales de la salud reconocidos tanto por la opinión pública como por el Estado. Una ventaja adicional de este enfoque es que permite prestar atención tanto al aspecto material del sistema de identificación profesional (títulos profesionales y listas que harían las veces de tempranas bases de datos) como a las prácticas de legitimación de 
dicho sistema (emisión de títulos, apelación a la fuerza pública) y las resistencias o alternativas ofrecidas a dicho sistema por parte de quienes fueron excluidos como por parte de los potenciales pacientes.

Si bien el tema de la profesionalización médica en Perú y América Latina ha sido ampliamente estudiado, es necesario revisitar dicho proceso para comprender la coexistencia y no necesariamente la mutua exclusión entre diversos médicos profesionales y sanadores, en especial, en un contexto en que la línea que separaba a unos de otros era difusa. En realidad, el rol de los sanadores fue más visible y relevante en el desarrollo del actual sistema de salud de lo que solemos considerar. Por ello, es necesario ir más allá de la dicotomía legal/ilegal o formal/informal sugeridas en las fuentes oficiales y reexaminar los argumentos con que se buscó contenerlos. La etiqueta de "impostor" -al igual que la de "charlatán"- esconde un amplio, flexible y heterogéneo grupo de practicantes, que van desde curanderos indígenas hasta doctores extranjeros más mediáticos.

Considerando todo ello, el presente artículo busca entender cómo y a través de qué estrategias los médicos profesionales en Perú intentaron diferenciarse de otros sanadores, en un momento en que los pacientes favorecían la eficacia del sanador por encima de los títulos profesionales. Los documentos no siempre son generosos en abundancia, por lo que hemos procedido a incorporar fuentes de diversos ámbitos, que incluyen documentos judiciales, prensa, cartas de sanadores a las autoridades y documentos oficiales de la Facultad de Medicina. En la medida en que las fuentes lo permiten, hemos buscado revertir el enfoque, poniendo en el centro a los sanadores, para así destacar su extraordinaria capacidad de adaptación y flexibilidad para operar en diversos escenarios.

Este trabajo está dividido en tres partes. En la primera, se estudia el proceso de profesionalización de la medicina en el siglo XIX, para luego analizar dos temas específicos en la segunda y la tercera parte, respectivamente: los mecanismos de identificación profesional implementados por la Facultad de Medicina y la usurpación de identidades médicas por algunos sanadores.

\section{PROFESIONALIZACIÓN DE LA MEDICINA EN PERÚ}

Durante el siglo XIX, en Perú, al igual que en otros países, se produjo un proceso de profesionalización de la medicina, caracterizado -según el historiador francés Matthew Ramsey- por una reorganización y consolidación de los médicos por sobre otros sanadores que ejercían la medicina sin contar con la autorización o título para ello ${ }^{(9)}$. En los últimos años, Diego Armus, Adrián López, Marcos Cueto, Steven Palmer e Irina Podgorny - por mencionar solo algunos autores - han realizado importantes contribuciones para sistematizar los trabajos que se han producido en América Latina en torno a la historia de la salud en la región, incluyendo temáticas como la profesionalización de la práctica médica y el pluralismo médico ${ }^{(10,11,12)}$. En este proceso, los académicos argentinos han tenido un rol clave en la expansión de la definición de medicina más allá de los discursos médicos académicos, para incorporar prácticas y creencias populares de sanación ${ }^{(13,14,15)}$. En cuanto a la profesionalización, trabajos como los de Ricardo González Leandri han sido un referente para comprender la construcción del monopolio de los médicos en "las artes de curar", influyendo en nuevas generaciones para estudiar dicha problemática en diversas partes del país y América Latina ${ }^{(16)}$.

La batalla contra los denominados "charlatanes" fue una de las principales transformaciones en el plano de la profesionalización que se vivió en Occidente, y que involucró a la sociedad civil y las asociaciones médicas que iban ganando poder y prestigio, especialmente durante la segunda mitad del siglo XIX ${ }^{(17,18)}$. Una de las características de la profesionalización de la medicina y de otras profesiones liberales fue que la licencia o título otorgado por una institución avalada por el Estado pasó a ser un artefacto y mecanismo fundamental para diferenciarse de los demás. 
Los médicos profesionales se comprometieron a erradicar a los competidores potenciales que operaban sin los respectivos diplomas y reforzar su estatus superior en el mercado medicinal. Muchos de estos sanadores gozaban de gran estima y prestigio entre sus pacientes, quienes se referían a ellos como "doctores". Sin embargo, los médicos profesionales comenzaron a utilizar categorías diferentes para referirse a ellos, nombrándolos de manera peyorativa como "charlatanes" o "impostores". En el mejor de los casos eran llamados "empíricos", un término más neutral para referirse a aquellos que poseían conocimiento y entrenamiento médico, pero no los títulos correspondientes.

En Perú, desde fines del período colonial se comienzan a observar las primeras transformaciones en el campo de la educación médica, que apuntaron a un mayor entrenamiento y estatus de los médicos por sobre otros practicantes. Una de las figuras más reconocidas en este período fue el médico e intelectual Hipólito Unanue, quien desde su posición de funcionario en el gobierno borbónico y, con posterioridad a la naciente República, impulsó reformas en la educación médica como la creación del Anfiteatro Anatómico y el Colegio de Medicina, siendo elegido primer director del Real Colegio de Medicina y Cirugía de San Fernando en $1815^{(19,20,21,22)}$. Las reformas apuntaron, entre otras cosas, a diferenciar a los médicos criollos, con entrenamiento en el Colegio de Medicina, de los cirujanos, la mayoría negros y mulatos, los cuales eran vistos en una posición inferior y percibidos como una amenaza a la profesión. El historiador José R. Jouve ha visibilizado el importante rol que cumplían dichos cirujanos y médicos afroperuanos en el paso de la colonia a la república, los cuales, pese a gozar de un gran prestigio entre la población, fueron deliberadamente excluidos del proyecto modernizador de Unanue ${ }^{(21)}$.

El proyecto de Unanue fue exitoso pues, después de la Independencia, los médicos ostentaron una posición prominente en la política de la sociedad peruana. El historiador Jorge Basadre estimó que los médicos ocuparon ocho escaños - de un total de 79- en la
Asamblea encargada de redactar la primera Constitución. Siempre según Basadre, los médicos ocuparon el cuarto lugar como grupo profesional, solo superados en número por los abogados con 28 integrantes, los eclesiásticos, con 26 ; y los comerciantes, con $9^{(23)}$. Sin embargo, la agitación política entre los años 1830 y 1850 , marcada por guerras regionales e internacionales, estancamiento económico e inestabilidad política, hizo que los médicos perdieran visibilidad en la arena política, al igual que otros grupos profesionales civiles.

Solo a fines de la década de 1840 y por las próximas dos décadas se alcanzaría cierta estabilidad política, social y económica, con una sucesión presidencial iniciada por Ramón Castilla, así como por los ingresos de la exportación de guano ${ }^{(24)}$. En 1851, el gobierno inició una reforma educacional que, en el campo de la medicina, estuvo marcada por la fundación de la Facultad de Medicina de Lima (también Ilamada San Fernando), institución heredera del Protomedicato. Al igual que esta institución colonial, la Facultad de Medicina fue encargada de vigilar el ejercicio de las profesiones sanitarias (médicos, parteras, flebótomos, y boticarios) y la enseñanza de dichas profesiones ${ }^{(25,26)}$. Si bien en las ciudades de Arequipa y Cuzco existían cátedras de medicina al momento de inaugurarse la Facultad de Medicina de Lima en 1856, su funcionamiento fue intermitente debido a la escasez de recursos técnicos y maestros dedicados a la enseñanza. Ya en 1876, un reglamento general de instrucción había excluido ambas ciudades de sus Facultades de Medicina ${ }^{(27)}$.

A diferencia de otras casas de estudios en América Latina, la Facultad fue la entidad que estuvo a cargo de las labores que en otros países desarrolló el Estado a través del Ministerio de Salud. La inexistencia de tal Ministerio en Perú hasta 1935 dejó a cargo del decano de la Facultad la fiscalización de los practicantes no licenciados, quien debía denunciar ante las autoridades la práctica ilegal de la medicina a nivel nacional. Asimismo, San Fernando fue el único lugar donde era posible estudiar medicina, puesto que la élite médica limeña asentada en la costa se opuso 
tenazmente a la apertura de otras casas de estudios médicos en regiones ${ }^{(28)}$. Si bien esta excesiva centralización ocasionó una escasez de médicos en las provincias, también facilitó el reconocimiento de médicos profesionales entre pares, puesto que la comunidad médica peruana compartía una identidad común: casi todos los médicos titulados habían pasado por las aulas de San Fernando y sus registros se custodiaban en el archivo de la institución.

\section{Identificación profesional}

La Facultad de Medicina se embarcó en la regulación de las actividades de la profesión médica en un momento en que se estaba produciendo una revolución en la identificación personal en Perú y en otros lugares de América Latina ${ }^{(29)}$. La transición de una sociedad cara-a-cara, donde la mayoría de las personas residían en áreas específicas y se conocían unas a otras, hacia otra caracterizada por una movilidad espacial intensa y poblaciones más grandes, llevó a la aparición de técnicas más sofisticadas para establecer y fijar las identidades de las personas. La policía había estado experimentando con nuevos métodos de identificación desde mediados del siglo XIX, como las huellas dactilares y fotos policiales. La obsesión por establecer identidades únicas abarcó también técnicas complejas, como la antropometría, que consistían en la medición detalladas de los cuerpos de prisioneros y sospechosos. Las estaciones de policía, las cárceles y cualquier otra instalación pública se convirtieron en laboratorios donde los funcionarios examinaron, midieron, clasificaron y fotografiaron cientos de personas antes de enviarlas a cumplir sus condenas o dejarlas en libertad ${ }^{(30)}$.

Pese a que estos nuevos métodos eran más precisos que las técnicas convencionales, se enfrentaban a dos grandes problemas. Primero, la tecnología utilizada para obtener y validar fotografías, medidas antropométricas y huellas dactilares no era del todo asequible para individuos y organizaciones profesionales. Incluso la propia policía tuvo problemas financieros para implementar y mantener un equipo adecuado a la vez que capacitar al personal especializado. En segundo lugar, la asociación de estas técnicas con perfiles delictivos impidió (o al menos retrasó) su apropiación por parte de individuos y organizaciones y una mayor expansión de la sociedad civil hasta principios del siglo $X^{(31)}$.

A nivel institucional, las organizaciones profesionales y las asociaciones civiles tomaron diversos caminos al momento de proteger la identidad de sus miembros y asegurar una identificación única para ellos. En el caso de Perú, el auge de las asociaciones profesionales tuvo lugar a mediados del siglo XIX, y estuvo vinculado al crecimiento de ocupaciones típicas de la clase media, muchas de estas aprendidas en aulas universitarias $^{(31)}$. El tamaño pequeño, en general, de dichas asociaciones locales favoreció las reuniones periódicas y garantizó una participación activa de sus miembros, reforzando la identificación grupal por medio del contacto visual y directo. Independientemente de su naturaleza (políticas, de beneficencia, sociales, etc.), estas entidades Ilamaban públicamente a reuniones a través de la prensa, facilitando así que sus miembros se conocieran y forjaran una identidad común. El rápido crecimiento demográfico de Lima, que pasó de 58.236 habitantes en 1836 a 105.167 en 1862, favoreció la expansión de dichas asociaciones y las prácticas de sociabilidad en espacios públicos y privados ${ }^{(32)}$.

Como señala Marcos Cueto, organizaciones profesionales como la Academia Libre de Medicina o la Sociedad Médica Unión Fernandina ayudaron a consolidar la autoridad y el prestigio de la profesión médica a través de un proceso de desplazamiento de otros practicantes locales. Estas organizaciones se vieron obligadas entonces a desarrollar mecanismos de seguridad e identificación que evitara la usurpación de la profesión por extraños ${ }^{(31)}$. Así, la Facultad de Medicina buscó instaurar un modelo de vigilancia marcado por su centralismo, donde los médicos pasaban por sus aulas y sus registros se conservaban en sus archivos, incluso si estos se trasladaban a otras partes del país. No obstante, este 
proyecto resultó muy difícil de llevar a cabo. Más allá del grupo compacto de médicos que se graduaron en San Fernando debidamente registrados en su base de datos, hubo un escenario vasto y nebuloso de practicantes cuyas identidades y acciones escaparon al control de la élite médica asentada en la capital. La Facultad de Medicina realizó un gran esfuerzo para contener la presencia de practicantes "ilegales" y reafirmar su hegemonía en el panorama médico. Para ello, la Facultad buscó vigilar a dos grupos en particular: los practicantes en el interior del país y los médicos extranjeros, especialmente, a través de la verificación de sus títulos médicos.

El principal obstáculo en la lucha de la Facultad de Medicina de Lima y, en especial, de su decano para erradicar la práctica de médicos no licenciados o "charlatanes" fue la carencia de un sistema actualizado para identificar a quienes ejercían (o no) legalmente en el país. La relativa flexibilidad de las instituciones médicas coloniales, así como los turbulentos años posteriores a la Independencia, permitieron a los practicantes informales ganar terreno hasta que la Facultad comenzó recién a abordar este problema. Para subsanar esta carencia de información, desde fines de la década de 1860, el Consejo de la Facultad de Medicina de Lima acordó la publicación de listas de médicos, farmacéuticos, dentistas, matronas y flebotómicos que contaban con autorización de la Facultad de Medicina de Lima ${ }^{(33)}$. La Facultad se comprometía a enviar ejemplares de estas listas a las autoridades políticas y sus delegados residentes en todo el país para que estos pudiesen ejercer control en la práctica de la medicina y farmacia en el territorio nacional ${ }^{(34)}$.

En un inicio, las listas fueron recibidas con entusiasmo por los delegados regionales y se consideraron un mecanismo fundamental en la lucha contra el charlatanismo. Sin embargo, el entusiasmo inicial pronto fue reemplazado por indiferencia, pues la Facultad dejó de enviar listas actualizadas a sus delegados por largos períodos, pese a sus reiteradas solicitudes ${ }^{(35)}$. En la correspondencia recibida por la Facultad se observa que los delegados informaron en repetidas ocasiones que la información contenida en esas listas era incompleta y desactualizada, impidiendo así el monitoreo efectivo de posibles infractores. Por ejemplo, en julio de 1869, el delegado de Cusco informó al decano de la Facultad de Medicina que dos de los médicos cuyos nombres aparecían en la lista habían fallecido hacía ya mucho tiempo ${ }^{(36)}$. De forma similar, el delegado de Arequipa comunicaba sobre el fallecimiento de siete médicos que estaban incluidos en la lista recibida desde la capital|(37). Fuera de la capital, la autoridad de la Facultad de Lima y de sus delegados disminuía de manera notoria, especialmente en ciudades y pueblos alejados de la costa peruana.

Este aparente ordenamiento y jerarquización del cuerpo médico contrastó con muchas fricciones existentes entre autoridades políticas y delegados regionales, quienes en múltiples instancias hicieron caso omiso a estas listas y reglamentaciones. Como indicaba el delegado en Huaraz, en 1867, las listas estaban disponibles en la ciudad. Pese a ello, el juez de primera instancia había decidido contratar, como médico del cuerpo de gendarmes, al curandero Guigeres, sin que se pudiera hacer nada para evitarlo(38).

La circulación de médicos y otros profesionales de salud que aseguraban tener título profesional, junto con la centralización de la información de profesionales fueron grandes obstáculos que los delegados en provincias debieron hacer frente a la hora de luchar contra el ejercicio ilegal de la medicina. Mientras que, en Lima, identificar a los profesionales sin título y denunciarlos ante las autoridades políticas en búsqueda de sanciones no fue un problema mayor durante el siglo XIX, los delegados regionales tenían menos recursos institucionales para ejecutar las directivas de la Facultad. Si los delegados albergaban dudas acerca de si algún practicante tenía autorización oficial, estos debían enviar un informe a Lima y esperar una respuesta sobre la validez de los títulos. Este procedimiento podía tomar meses antes de que el Consejo de la Facultad tomara una decisión y la comunicara desde la capital al interior del país. Un proceso burocrático que, en la mayoría 
de los casos negaba los títulos consultados, y que al momento de recibir la respuesta el presunto sospechoso se había mudado ya de la ciudad, estableciendo una nueva práctica en una jurisdicción diferente y atendiendo a nuevos pacientes.

En los primeros años de funcionamiento de la Facultad de Medicina, la poca acuciosidad de las listas se debía en parte a conflictos internos entre los médicos y otros profesionales de salud que estaban bajo su jurisdicción, especialmente, aquellos de farmacia. Antes de la refundación de la Facultad de Medicina en 1856, la Escuela de Farmacia era una entidad independiente de la de Medicina, sus autoridades establecían los planes de estudios y estaban a cargo de velar por el correcto funcionamiento de la profesión y de las farmacias y boticas del país. Sin embargo, los fundadores de la Facultad consideraban la farmacia como una ciencia auxiliar y procedieron a subordinarla a la Facultad de Medicina. No debería sorprender entonces que, en 1860, el decano Cayetano Heredia informara que Silverio Ferrer, exdirector de la Escuela de Farmacia, se negaba a entregar los materiales del archivo, razón por la cual desconocían las licencias que había expedido la Escuela hasta esa fecha, y que por esta razón era imposible realizar listas ${ }^{(39)}$.

Ante dicho desconocimiento, las autoridades tuvieron que recurrir a varias estrategias para poder saber quiénes poseían títulos validados por la Facultad, sobre todo en regiones. Por ejemplo, en 1864, el subprefecto de Tarapacá tuvo que publicar en los periódicos de la ciudad un llamado a todos los practicantes de la zona, informando que tenían diez días para acercarse y presentar sus credenciales. El Ilamado fue exitoso pues, si bien ninguno de los cuatro médicos que ejercían tenía título profesional, todos ellos solicitaron una prórroga para viajar a Lima y rendir los exámenes pertinentes ${ }^{(40)}$.

Como señalábamos anteriormente, la excesiva centralización de la educación médica en el país facilitó a la Facultad de Medicina en Lima identificar quiénes poseían títulos legalmente validados. Pero la mayor dificultad para dicha institución no provino necesariamente del interior del país, sino de reconocer la legalidad de títulos de médicos foráneos. En estos casos, la Facultad tuvo que recurrir en muchos casos a los cónsules en el extranjero para que solicitaran información sobre los documentos presentados. Si bien, en su gran mayoría, los títulos extranjeros contaban con todas las certificaciones, las denuncias que llegaban sobre falsificación no eran pocas. En diversas ocasiones, dichas denuncias respondieron más bien a conflictos entre colegas locales y foráneos. Una de las formas que los médicos criollos utilizaban para desacreditar curaciones o teorías de sus colegas extranjeros fue a través de la negación de la validez de sus títulos profesionales.

Eso fue lo que le sucedió al médico francés Abel Victorino Brandín, fundador de los Anales Medicales, primera revista médica de Perú de 1824. Brandín pronto se ganó la animosidad de médicos locales, pues en su revista proponía nuevas terapias para pacientes con enfermedades mentales, criticando de paso a médicos y autoridades locales. En un artículo anónimo publicado en el diario El Telégrafo de Lima en 1833, los autores del texto ponían en duda no solo los títulos médicos de Brandín, sino también su desempeño en actividades profesionales en las que supuestamente había participado. En respuesta, Brandín presentó una carta de la Legación y Consulado General de Francia en Perú, donde se certificaban sus diplomas en Medicina otorgados en París y Quito, así como la pertenencia a diversas sociedades profesionales. También presentó un certificado emitido por el Protomédico de Perú que lo autorizaba a ejercer la medicina en el país. Una vez que sus credenciales fueron acreditadas, sus detractores encontraron nuevos argumentos para cuestionarlo hasta conseguir finalmente que abandonara el país ${ }^{(41)}$.

Al parecer, la estrategia de cuestionar las credenciales de médicos extranjeros -ya sea de forma anónima o pública-, y con ello dañar su credibilidad, continuó hasta bien entrado el siglo XX. Durante una sesión extraordinaria de los miembros de la Facultad de Medicina llevada a cabo en abril de 1919, el decano informaba haber recibido una carta 
sin remitente en la cual se aseguraba que el diploma del doctor americano Carlyle Wothy no era auténtico. Ante dicha situación, la Facultad decidió investigar la denuncia, y recurrió al cónsul de Perú en EEUU para que acreditara la validez del documento. Luego de analizarlo, el consulado reconoció que era legal y que, por ende, el ciudadano estadounidense no tenía ningún problema para ejercer en el país(42).

\section{Usurpación profesional}

Como señalamos anteriormente, la identidad médica se basó en el título profesional, por lo que no debe extrañar que el primer artículo del reglamento de 1888 sobre el ejercicio de la medicina y farmacia señalara "nadie podrá ejercer ningún ramo de las ciencias médicas, si no está provisto de un diploma dado por la Facultad de Medicina"(43). Para los médicos agrupados en la Facultad de Medicina, el diploma tenía un gran valor simbólico, puesto que la visibilidad de dicho documento en un consultorio o farmacia acreditaba el estatus profesional y los distinguía de los "otros". A pocos años de inaugurarse la Facultad, el reglamento de esta institución establecía como obligatoria la exhibición de este documento. Así, en 1879, el decano Miguel E. de los Ríos recordaba a la Comisión Inspectora de Boticas que, para comprobar que los farmacéuticos estaban ejerciendo su profesión conforme a la ley, debían asegurarse de que estos expusieran el diploma de farmacéutico otorgado por la Facultad de Medicina de Lima en un lugar visible(44).

Muy al pesar de los San Fernandinos, muchos pacientes y autoridades políticas consideraban que los títulos médicos carecían de valor en sí, puesto que un historial de curaciones exitosas decía más de la eficacia de un doctor que un título universitario. Así lo demuestra el caso de Federico Bockenham, médico inglés quien fuera médico titular de la Provincia de Tarapacá entre 1859 y 1866, un cargo que dependía y era financiado por el Supremo Gobierno. Luego de varios años en el cargo, el delegado de la Facultad de
Medicina en dicha provincia, el doctor Coleman, descubrió que el médico titular no poseía diploma de médico. Coleman inició una campaña pública en 1866 para lograr la destitución de Bockenham, a pesar de que en sus palabras las autoridades locales miraban con indiferencia la idea de removerlo del cargo. Como le explicaba Coleman al decano, el apoyo de importantes personalidades locales a Bockenham se debía a que estaba muy arraigada en la "opinión pública la idea de que no se necesita para conseguir el título de médico no tanto el de la ciencia cuanto de los buenos empeños"(45).

El ejercicio de la farmacia y medicina por personas no tituladas era bastante habitual en el país. Muchos de quienes actuaban como médicos no ocultaban la carencia de un título universitario, e incluso no tenían problema en firmar documentos legales definiéndose a sí mismos como empíricos. A falta de médicos titulados en provincias, muchos de estos practicantes tomaron parte activa del sistema de justicia, actuando como peritos médicos en causas criminales, realizando autopsias e, incluso, firmando permisos a jueces que solicitaban ausentarse de sus labores, como lo ha analizado Pablo Whipple ${ }^{(46)}$. Por esta razón, la existencia de denuncias de usurpación médica en los documentos de la Facultad de Medicina resulta particularmente interesante, porque permite entender la dinámica fuera del ámbito legal y de la capital.

De las pocas causas documentadas existentes en los registros de la Facultad, es posible deducir que los farmacéuticos tendían a falsificar más sus títulos profesionales que los médicos. Una posible respuesta es que los farmacéuticos estaban sujetos a un mayor control por parte de la Facultad de Medicina, ya que anualmente la Comisión Inspectora de Boticas - dependiente de dicha casa de estudios- realizaba una visita a todos los establecimientos farmacéuticos para corroborar el estado de las medicinas y asegurarse que estuvieran adecuadamente administrados por un farmacéutico titulado. La visita tenía un costo monetario para el boticario, y significaba un ingreso nada despreciable para las arcas de la Facultad. En Lima, dichas visitas 
se realizaban de manera periódica, según acreditan los documentos de la Facultad.

Uno de los primeros casos de usurpación de identidad registrado por la Facultad de Medicina corresponde al del farmacéutico italiano, Carlos Andreotti, quien ejercía su profesión en la capital con la autorización de la Facultad de Medicina. Sin embargo, un profesor de dicha institución solicitó, en unas de las sesiones del concejo, que se revisase su expediente bajo la sospecha de haber presentado un título falso. Ante dicha acusación, Andreotti solicitó a tres personas que acudieran al consulado de Cerdeña para que ofrecieran una declaración jurada sobre su identidad y sus títulos. Pero las declaraciones no fueron del todo convincentes para el decano Cayetano Heredia, quien aconsejó que la Facultad debía dirigirse al Supremo Gobierno para averiguar si la Universidad de Turín había otorgado dicho título a Andreotti, o se trataba de una falsificación. Desconocemos el desenlace de este caso en particular, pero hay registros de muchos otros casos similares en la documentación de la Facultad de Medicina ${ }^{(47)}$.

El fraude no era fácil de comprobar, especialmente cuando supuestos médicos o farmacéuticos brindaban nombres de personas que efectivamente aparecían en los registros de la Facultad. Ante la inexistencia de un registro fotográfico y de un documento de identidad nacional -este solo apareció en 1931 y restringido a los que tenían derecho al voto-, determinar si la persona era realmente quien aparecía en los registros de la Facultad era casi imposible. Conocemos el caso de Manuel Rodríguez, farmacéutico, quien con un supuesto título de la Facultad acudió a la Alcaldía del Cusco a solicitar la apertura de una botica. El tal Rodríguez cometió un grave error, ya que entregó datos contradictorios sobre su identidad que hicieron sospechar a las autoridades locales, solicitando información a la Facultad en Lima antes de otorgar la licencia para la botica. El fraude quedó expuesto cuando el decano informó que, según los registros, efectivamente existía un farmacéutico llamado "Manuel Rodríguez", el cual ejercía legalmente en Lima.
Al ser consultado, el verdadero Manuel Rodríguez declaró que no había pisado Cusco en toda su vida ${ }^{(48)}$.

La prensa jugó un rol importante en la identificación de sanadores no licenciados. Los anuncios pagados en diversos diarios y revistas del país era una de las estrategias más utilizadas por médicos profesionales y no profesionales para atraer clientela. Los miembros de la Facultad constantemente controlaban la publicidad de los "charlatanes", y aquellos que no aparecían en los registros de la Facultad eran denunciados inmediatamente al subprefecto o al alcalde de Lima, con el objeto de hacer cerrar consultas médicas y boticas. En noviembre de 1918, Jorge Winkelmann, "profesor masajista diplomado en la Facultad de Medicina de Lima", solicitó al alcalde se le devolviese el dinero de una multa impuesta por el inspector de $\mathrm{Hi}$ giene por ejercicio ilegal de la medicina. EI inspector, quien actuó a petición del decano de la Facultad de Medicina, justificó la multa a través de un anuncio en la prensa, donde Winkelmann se presentaba como profesional de medicina prometiendo curar enfermedades y no solo como "masajista" (Figura 1). En este caso, la alcaldía procedió a la solicitud del decano. Sin embargo, no siempre los reclamos de la Facultad fueron atendidos por las autoridades políticas ${ }^{(49)}$.

No solo los farmacéuticos locales fueron víctimas de usurpación profesional. Los herbolarios chinos, también lo sufrieron de quienes quisieron aprovecharse de su repentina fama adquirida luego de la epidemia de fiebre amarilla de $1868^{(50,51)}$. En 1877, el herbolario Tam Jing publicó un aviso en el diario El Comercio, en el cual advertía a su clientela que su consulta no se encontraba en la calle Plumereros, sino en la de Unión. Esta declaración la realizaba porque había visto en el diario El Comercio un anuncio en el cual su nombre plagiado "con el propósito de especular con él", pero que a diferencia de sus anuncios, tenía problemas de redacción ${ }^{(52)}$. Sin embargo, los herbolarios chinos más importantes de la ciudad solucionaron de manera eficiente el tema de usurpación médica al incluir, desde la década de 1910, 


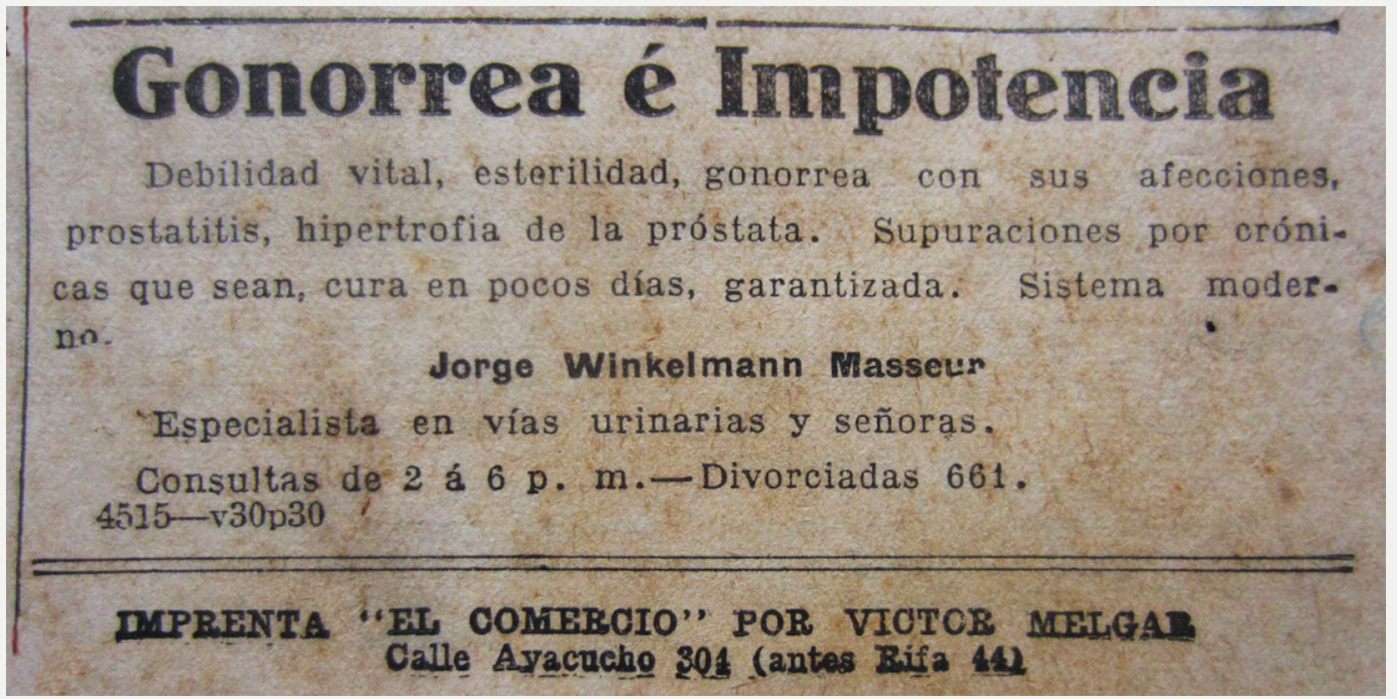

Figura 1. Anuncio de Jorge Winkelmann.

Archivo Histórico Municipalidad de Lima. Sección Higiene y Vacuna. Caja 3 (1914-1918)

fotografías personales en sus anuncios, permitiendo que los pacientes pudiesen reconocerlos $^{(50)}$. Se trataba de una técnica bastante innovadora, para un momento en que muchos retratos permanecían confinados a los estudios fotográficos y las cartas de visita.

Para los médicos de la Facultad de Medicina, la usurpación médica se agravaba cuando los "charlatanes" emulaban a los médicos profesionales a través de elementos que hasta ese momento habían sido utilizados exclusivamente por ellos, como símbolos de estatus que los identificaba como tales. Por ejemplo, los herbolarios chinos utilizaron técnicas de marketing en la prensa, y entregaban tarjetas de presentación y recetas médicas a sus pacientes similares a las ofrecidas por doctores profesionales. Incluso, como lo denunciaba un médico en 1906, uno de estos herbolarios había comprado un "coche médico", una especie de ambulancia en la cual visitaba a los enfermos "del mismo modo como lo hacen los médicos nacionales o extranjeros incorporados legalmente al gremio médico peruano" ${ }^{\prime(50)}$. Como se puede observar, de todos los no-profesionales que ejercían la medicina en el país, quienes más daño hicieron al proyecto de profesionalización fueron aquellos que adoptaron las mismas estrategias utilizadas por los médicos, convirtiéndose en los verdaderos "impostores".

\section{REFLEXIONES FINALES}

La Facultad de Medicina y los médicos profesionales que habían pasado por sus aulas desarrollaron una serie de campañas destinadas a erradicar a los sanadores no titulados, no solo como una manera de reforzar su identidad gremial, sino para desplazar a eventuales competidores. Esta institución buscó controlar a quienes ejercían en el campo de la salud, haciendo de la exhibición del diploma profesional un requisito obligatorio al igual que el registro en listas oficiales, las cuales eran enviadas a las autoridades médicas y políticas de todo el país. Diplomas y listas eran artefactos low-tech de identificación, alejados de la sofisticación de las técnicas que la policía estaba comenzando a incorporar por esos años, como las huellas digitales, la antropometría y las fichas individuales. No obstante, los diplomas y las listas fueron los antecedentes directos de nuestros modernos 
sistemas de identificación: los documentos de identidad y las bases de datos.

En el caso de Perú, los obstáculos para identificar a los doctores titulados de quienes no lo eran no reposaba necesariamente en lo avanzado de la tecnología de identificación disponible, sino en factores estructurales, como el centralismo y la creencia en que la medicina occidental bastaba en sí misma para imponerse frente a otros tipos de saberes. Las etiquetas que buscaban descalificar a los sanadores como "impostores" o "charlatanes" han impedido ver la diversidad de este grupo, así como el enorme repertorio de estrategias con las que contaban para sortear la vigilancia desde la capital. El caso de los herbolarios chinos es revelador, ya que supieron adaptarse a nuevas estrategias publicitarias y reducir la distancia con los médicos profesionales, a la vez que ampliar su clientela.

Como muestra este artículo, la división binaria entre doctores titulados y sanadores no titulados fue más compleja de lo esperado. En contra de lo que los médicos oficiales hubiesen esperado, los pacientes no llegaron a considerar a los sanadores no titulados como una amenaza para su salud, creando nuevos desafíos para la Facultad de Medicina. El "parecer" un doctor y tener un historial de "prácticas exitosas" fue en muchos casos suficiente y más importante para que los pacientes hicieran uso de sus servicios. Como hemos demostrado, la legitimidad de los sanadores no titulados (o la falta de esta) era producto de una negociación a nivel local y códigos de carácter social, muchas veces desconocidos para los médicos profesionales. La Facultad de Medicina privilegió las medidas punitivas antes que la incorporación o entrenamiento de sanadores no titulados en procedimientos occidentales o "modernos". Las consecuencias de estos desencuentros pueden explicar algunos problemas que experimentan los sistemas de salud en la actualidad y la tardía incorporación de saberes alternativos dentro de la oferta médica en países como Perú, con una larga tradición de prácticas tradicionales de salud.

La preferencia por la experiencia antes que por los títulos profesionales en sí mismos permite explicar la persistencia de "falsos médicos" hasta el día de hoy dado que, a pesar de los avances de la identificación profesional, personas como Malachi LoveRobinson, citado al inicio de este artículo, pueden ejercer públicamente la medicina sin ser detectados. Pese a que en la actualidad los pacientes tienen las herramientas para verificar las credenciales médicas a través de sitios web del Ministerio de Salud o en los respectivos Colegios Médicos, se hace muy poco uso de ellas; pues pareciera que, al igual que un siglo atrás, los títulos académicos tienen un rol secundario. Las investigaciones realizadas en los últimos años sobre Perú y América Latina han permitido una mayor visibilización y comprensión del rol de sanadores, curanderos y prácticas médicas más allá de las realizadas por los sistemas profesionales de salud.

El tema de los sanadores no titulados, su relación con los pacientes, el rol social dentro de determinadas comunidades, la manera en que absorbieron diferentes tipos de conocimientos, son áreas en las que nos queda un largo camino por recorrer. Es necesario reflexionar más a fondo sobre los conceptos que utilizamos en nuestras investigaciones para definir a estos sanadores no titulados. De esta manera, centrar nuestro foco de atención y revisitar los archivos y las fuentes -sobre todo las no institucionales- en los pacientes y en estos sanadores no titulados permitirá complejizar la historia de la salud que ha estado centrada en el discurso médico oficial y conocer más sobre cómo las personas desarrollaron un repertorio de terapias de salud fuera de aquellos espacios en donde las elites médicas tenían una mayor presencia. 


\section{REFERENCIAS BIBLIOGRÁFICAS}

1. Mosberger D. Accused fake 'teen Doctor' Malachi Love-Robinson arrested again. Huffington Post [Internet]. 3 mar 2016 [citado 15 dic 2018]. Disponible en: https://tinyurl.com/slmrleh.

2. Czubaj F. Crece el escándalo por los falsos médicos en hospitales y clínicas. La Nación [Internet]. 27 jul 2018 [citado 15 dec 2018]. Disponible en: https://tinyurl.com/rst4pzj.

3. Correa MJ, Zárate S. Historizar la profesión sanitaria: perspectivas desde Chile y Argentina. Dynamis. 2017;37(2):263-272.

4. Carrillo AM. Profesiones sanitarias y luchas de poderes en el México del siglo XIX. Asclepio. 1998;50(2):149-168.

5. Agostoni C. Médicos científicos y médicos ilícitos en la ciudad de México durante el Porfiriato. Estudios de Historia Moderna y Contemporánea de México. 1999;19:13-31.

6. Armus D. La enfermedad en la historiografía de América Latina moderna. Asclepio. 2002;54(2):4160.

7. Di Liscia MS. Reflexiones sobre la nueva historia social de la salud y la enfermedad en la Argentina. En: Carbonetti A, González Leandri L. Historias de salud y enfermedad en América Latina editors. Córdoba: Editorial CEA, UNC, CONICET; 2008. p. 15-47.

8. Armus D. Hybrid healers and ostracized health practitioners in early twentieth century Buenos Aires: The Pueyo case. En: The Gray Zones of Medicines(s): Towards a history of healers and healing in colonial and modern Latin America and the Caribbean; 14 sep 2018; Wisconsin-Madison.

9. Ramsey M. Professional and Popular Medicine in France, 1770-1830. Cambridge: Cambridge University Press; 1988.

10. Cueto M, Steven P. Medicine and Public Health in Latin America: A History. Cambridge: Cambrige University Press; 2015.

11. Armus, D, López, A. Disease, Medicine, and Health. En: Moya J, (ed.). The Oxford Handbook of Latin American History. New York: Oxford University Press; 2011. p. 424-453.

12. Podgorny I. Charlatans and Medicine in 19thCentury Latin America. Oxford Research Encyclopedia of Latin American History [Internet]. 29 mar
2017 [citado 10 sep 2019]. Disponible en: https:// tinyurl.com/smzmjct.

13. Di Liscia MS. Saberes, terapias y prácticas médicas en Argentina (1750-1910). Madrid: Consejo Superior de Investigaciones Científicas; 2002.

14. Álvarez A, Carbonetti A, (eds.). Saberes y prácticas médicas en la Argentina: un recorrido por historias de vida. Mar del Plata: Universidad Nacional de Mar del Plata; 2008.

15. Armus D, (ed.). Entre médicos y curanderos: Cultura, historia y enfermedad en la América Latina moderna. Buenos Aires: Editorial Norma; 2002.

16. González- Leandri R. Curar, persuadir, gobernar: La construcción histórica de la profesión médica en Buenos Aires, 1852-1886. Madrid: Consejo Superior de Investigaciones Científicas; 1999.

17. Porter R, (ed.). Patients and Practitioner: Lay perceptions of medicine in pre-industrial society. Cambridge: Cambridge University Press; 1986.

18. Porter R. Quacks: fakers and charlatans in English medicine. Stroud: Tempus; 2003.

19. Casalino C. Hipólito Unanue y la construcción del héroe: Análisis de la relación entre el Estado-nación y la sociedad peruana en su esfera cultural. Anales de la Facultad de Medicina. 2005;66:314-327.

20. Warren A. Medicine and politics in colonial Peru: Population growth and the Bourbon reforms. Pittsburgh: University of Pittsburgh Press; 2010.

21. Jouve-Martín J. The black Doctors of Colonial Lima: Science, race and writing in colonial and early Republican Peru. Montreal: McGill-Queen's University Press; 2014.

22. Arias-Schreiber J. Los médicos en la Independencia del Perú. Lima: P.L. Villanueva; 1971.

23. Basadre J. Elecciones y centralismo en el Perú (apuntes para un esquema histórico). Lima: CIUP, 1980.

24. McEvoy C. El legado castillista. Histórica. 1996; 20(2):211-241.

25. Lastres J. Historia de la medicina peruana. Vol. 3. La medicina en la República. Lima: Santa María; 1951.

26. Zavala J. El Protomedicato en el Perú: Del curanderismo empírico a la profesión. Lima: Universidad de San Martín de Porres, Facultad de Medicina Humana; 2008. 
27. Arias-Schreiber J. La Facultad de Medicina de Arequipa en el siglo XIX. Lima: Editorial Universitaria; 1973.

28. Palma P. "Science Can't Save Me": Public Health, Professional Medicine, and Medical Pluralism in Peru (1856-1935) [dissertation]. California: University of California; 2017.

29. Ragas J. Documenting hierarchies: State building, identification and citizenship in modern Peru [dissertation]. California: University of California, Davis; 2015.

30. García Ferrari M. Ladrones conocidos/Sospechosos reservados. Identificación policial en Buenos Aires, 1880-1905. Buenos Aires: Prometeo; 2010.

31. Cueto M. Excelencia científica en la periferia: actividades científicas e investigación biomédica en el Perú 1890-1950. Lima: Consejo Nacional de Ciencia y Tecnología; 1989.

32. Gootenberg P. Población y etnicidad en el Perú republicano (siglo XIX): algunas revisiones. Lima: Instituo de Estudios Peruanos; 1995.

33. De los Ríos M. Carta a: Apoderados de la Facultad. (Lima). 15 jul 1869. Documento alojado en: Notas e Informes, Archivo Histórico de la Facultad de Medicina de Lima.

34. De los Ríos M. Carta a: Fiscal General. (Lima). 18 jul 1866. Documento alojado en: Notas e Informes, Archivo Histórico de la Facultad de Medicina de Lima.

35. Apoderado de la Facultad en la Provincia de Chincha. Carta a: Decano de la Facultad (Chincha). 31 jul 1869. Documento alojado en: Documentos recibidos, Archivo Histórico de la Facultad de Medicina de Lima.

36. Apoderado de la Facultad en la Provincia de Cusco. Carta a: Decano de la Facultad (Cusco). 17 jul 1869. Documento alojado en: Documentos recibidos, Archivo Histórico de la Facultad de Medicina de Lima.

37. Apoderado de la Facultad en la Provincia de Arequipa. Carta a: Decano de la Facultad (Arequipa). 17 nov 1870. Documento alojado en: Documentos recibidos, Archivo Histórico de la Facultad de Medicina de Lima.

38. Apoderado de la Facultad en la Provincia de Huaraz. Carta a: Decano de la Facultad (Huaraz). 20 oct 1867 . Documento alojado en: Documentos recibidos, Archivo Histórico de la Facultad de Medicina de Lima.
39. Heredia C. Carta a: Ministro de Instrucción y Beneficencia Pública (Lima). 23 ago 1860. Documento alojado en: Notas e Informes, Archivo Histórico de la Facultad de Medicina de Lima.

40. Delegado de la Provincia de Tarapacá. Carta a: Sub Prefecto de la Provincia de Tarapacá (Iquique). 28 jan 1864. Documento alojado en: Documentos enviados, Archivo Histórico de la Facultad de Medicina de Lima.

41. Paz Soldán CE. La vida aventurera de Abel Victorino Brandin, el introductor del sulfato de quinina en la América Meridional. Anales de la Sociedad Peruana de Historia de la Medicina. 1940;2:10-29.

42. Consejo de la Facultad de Medicina. Sesión extraordinaria. 1919. Documento alojado en: Actas del Consejo, Archivo Histórico de la Facultad de Medicina de Lima.

43. Reglamento del ejercicio de la Medicina y de la Farmacia. 28 nov 1888. Documento alojado en: Archivo Digital de la Legislación del Perú. Disponible en: https://tinyurl.com/s9dwrqf.

44. De los Rios M. Carta a: Inspección de Higiene del Consejo Municipal (Lima). 1879 May 8. Documento alojado en: Higiene y Vacuna Box 2 (1870-1884), Archivo Histórico de la Municipalidad de Lima.

45. Apoderado de la Facultad en la Provincia de Tarapacá. Carta a: Decano de la Facultad (Iquique). 29 abr 1866. Documento alojado en: Documentos recibidos, Archivo Histórico de la Facultad de Medicina de Lima.

46. Whipple P. Carencias materiales, respetabilidad y prácticas judiciales en Perú durante los inicios de la República. Historia Crítica. 2013;49:55-79.

47. De los Ríos M. Carta a: Ministro de Instrucción Pública (Lima). 11 jun 1860. Documento alojado en: Notas e Informes, Archivo Histórico de la Facultad de Medicina de Lima.

48. Odriozola M. Carta a: Delegado de Cusco (Lima). 8 may 1879. Documento alojado en: Notas e Informes, Archivo Histórico de la Facultad de Medicina de Lima.

49. Expediente seguido por Jorge Wilkeman (Lima). 18 nov 1918. Documento alojado en: Higiene y Vacuna Box 3 (1914-1918), Archivo Histórico de la Facultad de Medicina de Lima.

50. Palma P. Sanadores inesperados: medicina china en la era de migración global (Lima y California, 1850-1930). História, Ciências, Saúde Manguinhos. 2018;25(1):13-31. 
51. Palma M, Ragas J. The "Other Plague": Chinese Healers and their Patients in Lima. Paper presented at: The Gray Zones of Medicines(s): Towards a History of Healers and Healing in Colonial and Modern Latin America and the Caribbean; 14 sep 2018; Wisconsin-Madison.
52. Jing T. Aviso importante. El Comercio (Lima). 2 oct 1877.

\section{FORMA DE CITAR}

Palma P, Ragas J. Desenmascarando a los impostores: Los médicos profesionales y su lucha contra los falsos médicos en Perú. Salud Colectiva. 2019;15:e2162. doi: 10.18294/sc.2019.2162.

Recibido: 4 de febrero de 2019 | Versión final: 14 de octubre de 2019 | Aprobado: 6 de noviembre de 2019

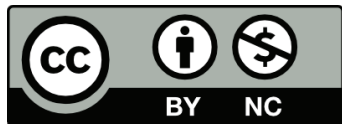

http://dx.doi.org/10.18294/sc.2019.2162 